Original article

\title{
PREVALENCE OF LINGUATULA SERRATA NYMPHS AND PATHOLOGICAL LESIONS OF INFECTED MESENTERIC LYMPH NODES AMONG RUMINANTS IN KERMANSHAH, WESTERN IRAN
}

\author{
M. HASHEMNIA ${ }^{1}$, F. REZAEI $^{1}$, M. SAYADPOUR ${ }^{1} \&$ Y. SHAHBAZI ${ }^{2}$ \\ ${ }^{1}$ Department of Pathobiology, ${ }^{2}$ Department of Food Hygiene and Quality \\ Control; Veterinary Medicine Faculty, Razi University, Kermanshah, Iran
}

\begin{abstract}
Summary
Hashemnia, M., F. Rezaei, M. Sayadpour \& Y. Shahbazi, 2018. Prevalence of Linguatula serrata nymphs and pathological lesions of infected mesenteric lymph nodes among ruminants in Kermanshah, western Iran. Bulg. J. Vet. Med., 21, No 1, 94-102.

Linguatulosis is one of the important zoonotic diseases that cause public health problems worldwide. This study was carried out from March 2014 to February 2015 to estimate the prevalence of nymphal stages of Linguatula serrata in ruminants of different sex and age slaughtered at the Kermanshah municipal abattoir, in western Iran. For this purpose, the mesenteric lymph nodes (MLNs) were examined grossly and three MLNs for each animal were collected for parasitological and pathological studies. Of the total of 456 sheep (252 $\delta$ and $204 \%$ ), 390 goats (215 $\delta$ and 175 + $)$ and 412 cattle (255 and 157 + $), 90$ (19.7\%), 98 (25.1\%) and 53 (12.8\%) were infected with nymph stages of $L$. serrata, respectively. A significant difference in the prevalence of linguatulosis was observed among studied animals $(\mathrm{P}<0.05)$ and the highest and lowest prevalence were detected in goats and cattle, respectively. Prevalence of $L$. serrata nymphs in males and females was not significantly different $(\mathrm{P}>0.05)$. The prevalence increased with age $(\mathrm{P}<0.05)$. Grossly, the infected MLNs were enlarged, and their cross-sectional areas were coarse, firm, granulated, oedematous, and red in colour. Necrosis, oedema, haemorrhages, depletion of the lymphocytes and granulomatous reactions which are characterised by infiltration of mononuclear inflammatory cell around the parasite were the main histopathological lesions. These results indicated the high prevalence of $L$. serrata infection in livestock animals in Kermanshah. Furthermore, this study provides a preliminary baseline data for the future monitoring of this potentially important parasitic disease. The potential importance of these findings to human health is discussed.
\end{abstract}

Key words: histopathology, Iran, Linguatula serrata, lymph nodes, prevalence, ruminants

\section{INTRODUCTION}

Linguatula serrata (Pentastomum denticulatum) commonly known as 'tongue worm' is an aberrant arthropod of the class Pentastomida (Hendrix, 1998). A wide range of mammals is intermediate hosts for L. serrata, but herbivores such as cattle, goats, sheep, camels and other ruminants are the best hosts for develop- 
ment of parasite's nymphal stages (AlcalaCanto et al., 2007). Infective eggs containing fully developed larvae are discharged into the environment by nasopharyngeal secretions of carnivorous mammals and are ingested by the intermediate hosts in which it develops to the nymphal stage in mesenteric lymph nodes, liver, lung, etc. (Berger \& Marr, 2006). Final hosts such as dogs become infected by eating the infected visceral organs of the herbivorous hosts (Soulsby, 1982).

In humans, two forms of linguatulosis have been identified: nasopharyngeal linguatulosis, known also as Halzoun or Marrara syndrome, is often produced following consumption of raw or undercooked infected viscera (liver, lung and lymph nodes) of infected animals (Rezaei et al., 2012) and visceral linguatulosis following consumption of contaminated water, vegetables, or fruits with the eggs (Yao et al., 2008; Tappe \& Büttner, 2009).

Most of the human cases of linguatulosis, especially those with the visceral form, remain undetected because this disease is usually asymptomatic and the clinical features are nonspecific and, more importantly, necropsy of humans is not easily permitted in many countries including Iran. Despite all these limitations, many cases of linguatulosis have previously been reported in this country (Sadjjadi et al., 1998; Maleky, 2001; Rokni, 2008).

However, several studies have been carried out as regards the prevalence of $L$. serrata infection in animals including, dogs (Meshgi \& Asgarian, 2003; Oryan et al., 2008), camels (Pourjafar et al., 2007; Tajik et al., 2007; Shakerian et al., 2008; Oryan et al., 2011), buffaloes (Tajik et al., 2008; Rezaei et al., 2011), sheep (Shekarforoush et al., 2004; Tavassoli et al., 2007; Nourollahi Fard et al., 2011), cattle (Hami et al., 2009; Nourollahi Fard et al., 2010a) and goats (Razavi et al., 2004; Nourollahi Fard et al., 2010b) in many parts of Iran, but the incidence of $L$. serrata in herbivores in western Iran is practically unknown.

In view of the high prevalence of linguatulosis in domestic animals and the number of reports of human infestation in recent years from Iran, the present study was designed to investigate the prevalence and pathology of L. serrata among ruminants slaughtered at Kermanshah slaughterhouse, Kermanshah Province, western Iran.

\section{MATERIALS AND METHODS}

During an one-year period (March 2014 to February 2015), 456 sheep (252 $\delta$ and 204 ㅇ), 390 goats (215 $\delta$ and 175 우) and 412 cattle (255 $\delta$ and 157 9 ) in four age groups $(<1,1-2,2-3$, and $>3$ years old $)$ were selected randomly at the Kermanshah slaughterhouse. For this study, mesenteric lymph nodes were examined grossly for pathological alterations and three MLNs for each animal were collected in PBS and transferred to the Parasitology Department of Razi University. After recording the gross appearance, each lymph node was cut longitudinally, put in Petri dishes containing normal saline and examined under a dissecting microscope for $L$. serrata nymphs.

All mesenteric lymph nodes containing immature stages of $L$. serrata were fixed in $10 \%$ neutral buffered formalin, dehydrated in graded ethanol, and embedded in paraffin. Sections of $5 \mu \mathrm{m}$ in thickness were stained with haematoxylin and eosin and examined by light microscopy.

The chi-square test was used for comparison of the prevalence of linguatulosis between males and females and the different age groups. Differences were considered significant when $\mathrm{P}<0.05$, using com- 
Prevalence of Linguatula serrata nymphs and pathological lesions of infected mesenteric lymph nodes...

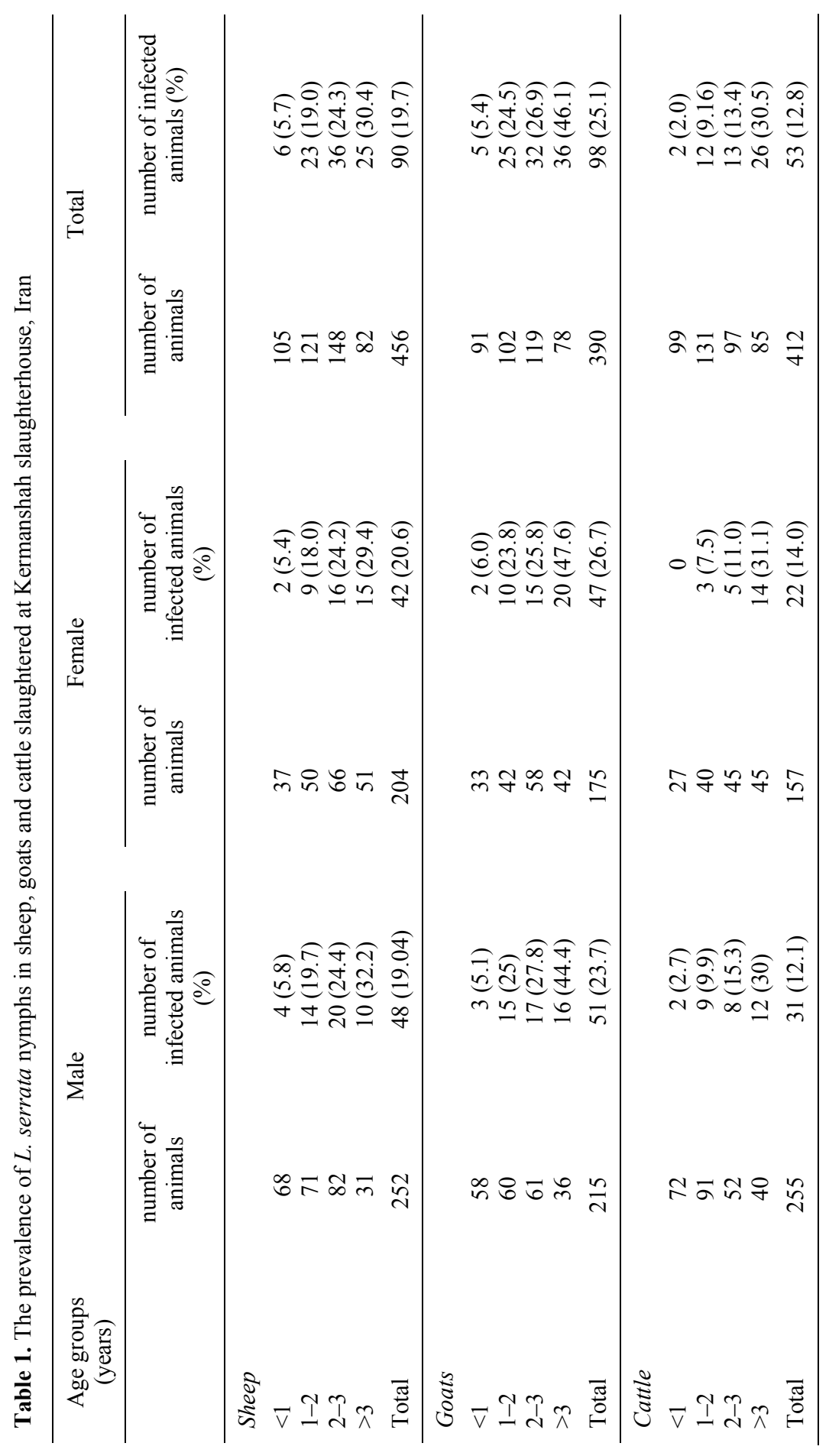


puter software SPSS version 16 for Windows (SPSS, Chicago, IL, USA).

\section{RESULTS}

The prevalence of $L$. serrata nymphs in mesenteric lymph nodes of 452 sheep, 390 goats and 412 cattle slaughtered at Kermanshah slaughterhouse, Iran, divided into different sex and age groups is summarised in Table 1.

Ninety out of $456(19.7 \%)$ sheep (19.04\% $\delta$ and 20.06 \%), 98 out of 390 (25.1\%) goats $(23.7 \%$ and $26.7 \%$ o $)$ and 53 out of $412(12.8 \%)$ cattle $(12.1 \%$ $\widehat{\delta}$ and $14 \%$ O) were infected with nymphal stages of L. serrata. Although a difference was seen in the prevalence between males and females in all animals, it was not significant at the same age groups of males and females. The results showed a higher infection rate of mesenteric lymph nodes in goats compared with sheep and cattle. However, this difference was only significant between goats and cattle $(\mathrm{P}<0.05)$. The prevalence increased with age in all animals $(\mathrm{P}<0.05)$.

The gross appearance of mesenteric lymph nodes consisted of haemorrhagic and necrotic lesions. The infected MLNs were enlarged, and their cross-sectional areas were coarse, firm, granulated, oedematous, and red in colour. Most of the affected lymph nodes contained nymphal L. serrate (Fig. 1)

Histopathologically, the nymphs of $L$. serrate were elongated and their cuticles showed transverse striations with rows of downward oriented small spines (Fig. 2). They were observed within oedematous cyst-like spaces surrounded by a wall composed of fine fibrous and dense lymphocytic zones. Acute lesions consisted of traumatic foci, with haemorrhages and oedema and massive tissue necrosis.
Haemosiderosis by infiltration of haemosiderophages was usually observed in these sections (Fig. 3).

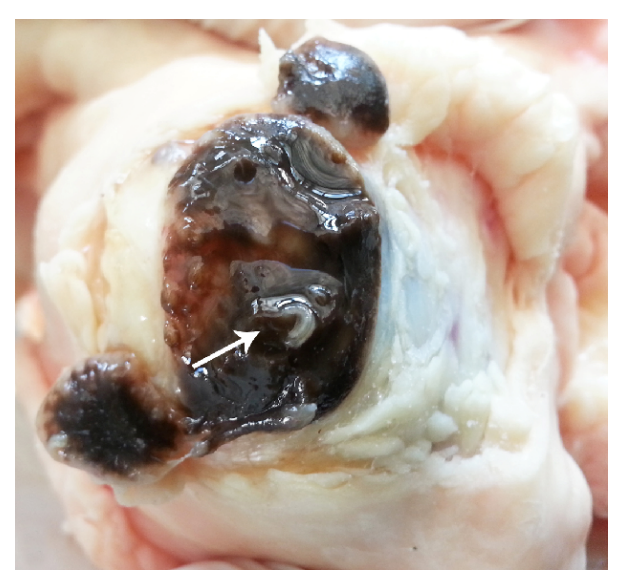

Fig. 1. The nymphal stages of L. serrata in an enlarged, firm and oedematous mesenteric lymph node (arrow).

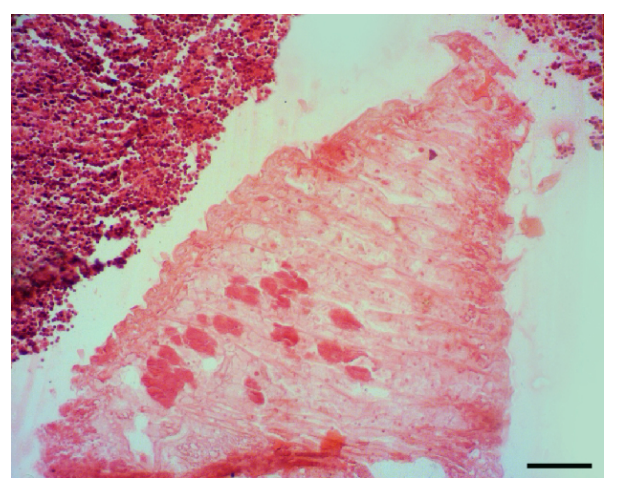

Fig. 2. Posterior part of the L. serrata nymph with segmented body and transversely striated spines in the mesenteric lymph node ( $\mathrm{H}$ and $\mathrm{E}$, scale bar $=80 \mu \mathrm{m}$ )

In older lesions, dead larvae were surrounded by chronic granulomatous inflammation, characterised by accumulation of epithelioid macrophages, giant multinucleated cells, lymphocytes and plasma cells around the necrotic areas (Fig. 4, 5). Fibrosis of the infected lymph 


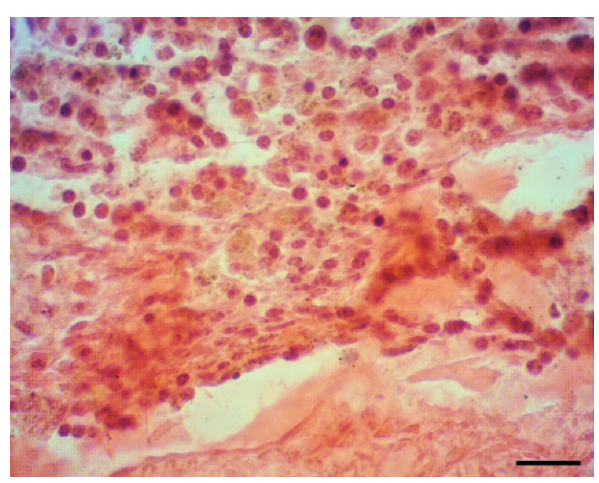

Fig. 3. Cross-sectional area of an infected mesenteric lymph node infected with $L$. serrata nymphs. Haemosiderophages infiltration and oedematous changes in the lymph node are evident $(\mathrm{H}$ and $\mathrm{E}$, scale bar $=20 \mu \mathrm{m})$.

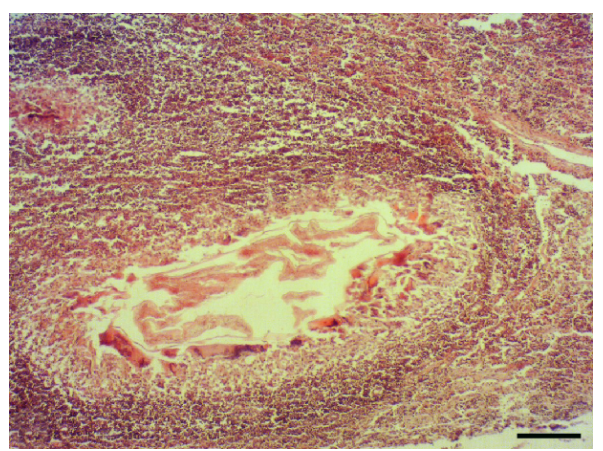

Fig. 4. Cross section of L. serrata nymphs in the mesenteric lymph node. The dead larva is surrounded by chronic granulomatous inflammation ( $\mathrm{H}$ and $\mathrm{E}$, scale bar $=100 \mu \mathrm{m})$.

nodes was usually evident around the parasite and encapsulated it from the surrounding areas. In some cases, cell population of lymph nodes was decreased, particularly in the outer cortical region and partly substituted by oedema.

\section{DISCUSSION}

Several studies have been conducted in Iran to determine the prevalence of linguatulosis in ruminants. The prevalence

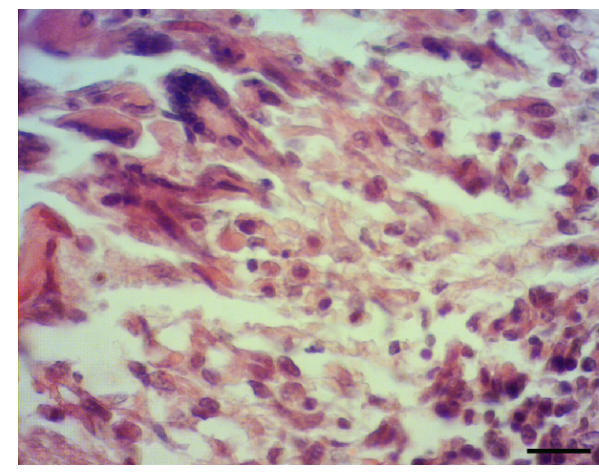

Fig. 5. Cross-sectional area of an infected mesenteric lymph node infected with $L$. serrata nymphs. Accumulation of epithelioid macrophages, giant multinucleated cells, lymphocytes and plasma cells around the parasite ( $\mathrm{H}$ and $\mathrm{E}$, scale bar $=20 \mu \mathrm{m})$.

rates of $11.5 \%, 52 \%$ and $16.1 \%$ linguatulosis in sheep have been reported from Fars, Kerman and west Azerbaijan provinces of Iran, respectively (Shekarforoush et al., 2004; Tavassoli et al., 2007; Nourollahi Fard et al., 2011). Razavi et al. (2004) and Nourollahi Fard et al., (2010b) reported a prevalence of $29 \%$ and $49.1 \%$ linguatulosis in goats from Shiraz and Kerman, eastern Iran. Prevalence of $16.4 \%$ in cattle in Urmia western Iran (Tajik et al., 2006), 16.22\% in Kerman eastern Iran (Nourollahi Fard et al., 2010a), 14.8\% in Babol northern Iran (Youssefi \& Hadizadeh Moalem, 2010), and $0.25 \%$ in Tabriz, western Iran (Hami et al., 2009) have previously been reported.

This parasitic infection is also endemic in some other parts of the world. A prevalence of $21 \%$ in cattle, $19 \%$ in goats and $5.19 \%$ in sheep have been recorded in South India and Turkey, respectively (Ravindran et al., 2008; Gül et al., 2009).

In this study, $19.7 \%$ of the sheep, $25.1 \%$ of the goats and $12.8 \%$ of the cattle had L. serrata nymphs in their MLNs. The 
difference between the prevalence of linguatulosis in Kermanshah with other regions of Iran may be due to a regional difference in distribution of parasite.

In the present study, the highest prevalence of linguatulosis was observed in goats, while the lowest prevalence recorded in cattle. These differences may be due to the grazing habits of the animal species and also differing forage habitat and herbaceous foods. Additionally, exposure or proximity to definitive hosts (especially dogs) and resultant parasite eggs shed into the environment may have an important role.

The prevalence of $L$. serrata nymphs in females was higher than in males in all animal species, but these differences were not statistically significant similarly to those reported previously by other researchers (Nourollahi Fard et al., 2011; Oryan et al., 2011). In the study conducted by Shekarforoush et al., (2004), the prevalence of $L$. serrata nymphs has been significantly greater in female than in male sheep. The differences in the results of the various studies may be due to differences in the management and grazing systems and in the number of the animals under study.

According to the results, the prevalence increased with age and was highly significant. The lower prevalence in the younger groups may due to the type of feeding (milk instead of grass) and also to lesser contact to the definite host (Nourollahi Fard et al., 2011).

In this study, the main histopathological lesions included necrosis, oedema, haemorrhages, depletion of the lymphocytes and granulomatous reactions characterised by infiltration of mononuclear inflammatory cells around the parasite. These findings were consistent with the lesions reported by other investigators in sheep and camel (Miclãuş et al., 2008; Oryan et al., 2011).

According to these lesions, it seems that the parasite can influence both the humeral and cell-mediated immune systems of the intermediate hosts and make the infected animals or humans susceptible to other infective diseases or neoplastic changes.

Recently, Mir et al. (2009) reported the concurrent occurrence of visceral linguatulosis with paratuberculosis in alpine cross goats. The pathogenicity of Crohn's and tuberculosis diseases in humans is similar to paratuberculosis in livestock animals, and infection with the visceral linguatulosis may predispose to these or other diseases.

The high prevalence of $L$. serrata in this study shows that Kermanshah is an endemic or a hyperendemic area for linguatulosis. However, this result was based upon the examination of only histopathological sections from only three mesenteric lymph nodes per animal. Definitely, the prevalence would possibly have been higher, if more lymph nodes and also samples from the liver, spleen, and other visceral organs were examined.

The major reason for this high prevalence of linguatulosis in this area is the close contact between the dogs and other carnivores and various domestic animals in most household livestock units that may favor the contamination of feed and water intended for livestock with eggs of $L$. serrata through excreta of dogs.

On the other hand, the offal or carcasses of infected domestic animals are the principal source of infection of dogs and the ingestion of raw viscera of herbivores results in the high prevalence of linguatulosis in dogs and create excellent conditions for the maintenance of parasite in the environment (Oryan et al., 2008). 
Prevalence of Linguatula serrata nymphs and pathological lesions of infected mesenteric lymph nodes ...

Humans can be infected with both adult and immature forms of $L$. serrata acting as final and intermediate hosts. Although the larval or nymphal infection in herbivores is usually clinically asymptomatic, the infected animals are considered to be the main source of infection for humans (Oryan et al., 2011). Therefore, the high prevalence rate of $L$. serrata infection in lymph nodes of ruminants in this area should be considered as an important risk factor for human being infection.

People consuming livestock visceral organs as raw or semi-cooked meal could potentially acquire the parasite. In addition, consumption of raw or undercooked liver by pregnant mothers because of its high iron and vitamin content is very common in Iran and leads to an increased prevalence of linguatulosis in the human population (Nourollahi Fard et al., 2011).

\section{CONCLUSION}

This study showed a high spread of $L$. serrata infection in livestock animals in Kermanshah. The high prevalence of infection in ruminants is of concern owing to the zoonotic nature of the parasite and the risk of infection to humans and other animals. Although the diagnosis of linguatulosis is difficult, it should not be ignored; adopting proper and reliable diagnostic methods to detect infection in slaughterhouses, restrict backyard slaughter, enforce legislation on meat inspection and improve veterinary services can help to control and prevention of the disease in this area. Furthermore, public health education about the disease is highly recommended to the community.

\section{ACKNOWLEDGEMENTS}

The authors would like to thank the authorities of Veterinary School, Kermanshah University for their financial support and cooperation.

\section{REFERENCES}

Alcala-Canto, Y., A. Alberti-Navarro \& F. Ibbara-Velarde, 2007. Serin protease activity demonstrated in larval stage of the Pentastomid Linguatula serrata. Parasitology Research, 100, 1011-1014.

Berger, S. A. \& J. S. Marr, 2006. Human Parasitic Diseases Sourcebook, Jones \& Bartlett, Sudbury, p. 294.

Gül, A., S. Değer \& V. Denizhan, 2009. The prevalence of Linguatula serrata nymphs in sheep in the Van Province. Türkiye Parazitoloji Dergisi, 33, 25-27.

Hami, M., S. R. Naddaf, I. Mobedi, M. ZareBidaki, S.S. Athari, B. Hajimohammadi \& G. Anaraki-Mohammadi, 2009. Prevalence of Linguatula serrata infection in domestic bovids slaughtered in Tabriz Abattoir, Iran. Iranian Journal of Parasitology, 4, 25-31.

Hendrix, C. M., 1998. Diagnostic Veterinary Parasitology. Mosby Inc, pp. 228-233.

Maleky, F., 2001. A case report of Linguatula serrata in human throat from Tehran, central Iran. Indian Journal of Medical Sciences, 55, 439-441.

Meshgi, B. \& O. Asgarian, 2003. Prevalence of Linguatula serrata infestation in stray dogs of Shahrekord, Iran. Journal of Veterinary Medicine B, 50, 466-467.

Miclãuş, V., A.D. Mihalca, O. Negrea \& L. Oanã, 2008. Histological evidence for inoculative action of immature Linguatula serrata in lymph nodes of intermediate host. Parasitology Research, 102, 13851387.

Mir, M. S., M. M. Darzi, I. Hussain \& S. A. Wani, 2009. Concurrent occurrence of visceral linguatulosis and paratuberculosis in 
alpine cross goats (Capra hircus). Veterinarski Arhiv, 79, 301-314.

Nourollahi Fard, S. R., R. Kheirandish, E. Norouzi Asl \& S. Fathi, 2010a. The prevalence of Linguatula serrata nymphs in mesenteric lymph nodes in cattle. American Journal of Animal and Veterinary Sciences, 5, 155-158.

Nourollahi Fard, S.R., R. Kheirandish, E. Norouzi Asl \& S. Fathi, 2010b. The prevalence of Linguatula serrata nymphs in goats slaughtered in Kerman slaughterhouse, Kerman, Iran. Veterinary Parasitology, 171, 176-178.

Nourollahi Fard, S. R., R. Kheirandish, E. Norouzi Asl \& S. Fathi, 2011. Mesenteric and mediastinal lymph node infection with Linguatula serrata nymphs in sheep slaughtered in Kerman slaughterhouse, southeast Iran. Tropical Animal Health and Production, 43, 1-3.

Oryan, A., S. M. Sadjjadi, D. Mehrabani \& M. Rezaei, 2008. The status of Linguatula serrata infection of stray dogs in Shiraz,Iran. Comparative Clinical Pathology, 17, 55-60.

Oryan, A., M. Khordadmehr \& V. R. Ranjbar, 2011. Prevalence, biology, pathology, and public health importance of linguatulosis of camel in Iran. Tropical Animal Health and Production, 43, 1225-1231.

Pourjafar, M., H. Azizi \& S. Darabi, 2007. The prevalence of nymphal stage of Linguatula serrata in camels (Camelus dromedaries) in Najaf-Abad, Iran. Journal of Camel Practice and Research, 14, 171173.

Ravindran, R., B. Lakshmanan, C. Ravishankar \& H. Subramanian, 2008. Prevalence of Linguatula serrata in domestic ruminants in South India. Southeast Asian Journal of Tropical Medicine \& Public Health, 39, 808-812.

Razavi, S. M., S. S. Shekarforoush \& M. Izadi, 2004. Prevalence of Linguatula serrata nymph in goats in Shiraz, Iran. Small Ruminant Research, 54, 213-217.
Rezaei, F., M. Tavassoli \& A. Mahmoudian, 2011. Prevalence of Linguatula serrata infection among dogs (definitive host) and domestic ruminants (intermediate host) in the North West of Iran. Veterinarni Medicina, 56, 561-567.

Rezaei, F., M. Tavassoli \& M. Javdani, 2012. Prevalence and morphological characterizations of Linguatula serrata nymphs in camels in Isfahan Province, Iran. Veterinary Research Forum, 3, 61-65.

Rokni, M. B., 2008. The present status of human helminthic diseases in Iran. Annals of Tropical Medicine and Parasitology, 102, 283-295.

Sadjjadi, S. M., S. M. Ardehali \& A. Shojaei, 1998. A case report of Linguatula serrata in human pharynx from Shiraz, southern Iran. Medical Journal of The Islamic Republic of Iran, 12, 193-194.

Shakerian, A., S. S. Shekarforoush \& H. Ghafari-Rad, 2008. Prevalence of Linguatula serrata nymphs in Najaf-Abad, Iran. Research in Veterinary Science, 44, 243245.

Shekarforoush, S. S., S. M. Razavi \& M. Izadi, 2004. Prevalence of Linguatula serrata nymphs in sheep in Shiraz, Iran. Small Ruminant Research, 52, 99-101.

Soulsby, E. J. L., 1982. Helminths, Arthropods and Protozoa of Domesticated Animals. $7^{\text {th }}$ edn, Baillière Tindall, London, pp. 497498.

Tajik, H., M. Tavassoli, B. Dalir-Naghadeh \& M. Danehloipour, 2006. Mesenteric lymph nodes infection with Linguatula serrata nymphs in cattle. Iranian Journal of Veterinary Research, 7, 82-85

Tajik, H., M. Tavassoli \& H. Khani, 2007. Prevalence of Linguatula serrata nymphs in slaughtered camels of Iran. Journal of Camel Practice and Research, 14, 69-71.

Tajik, H., M. Tavassoli, S. Javadi \& H. Baghebani, 2008. The prevalence rate of Linguatula serrata nymphs in Iranian river buffaloes. Asian Journal of Animal and Veterinary Advances, 3, 174-178 . 
Prevalence of Linguatula serrata nymphs and pathological lesions of infected mesenteric lymph nodes ...

Tappe, D. \& D. W. Büttner, 2009. Diagnosis of human visceral pentastomiasis. PLOS Neglected Tropical Diseases, 3, e320.

Tavassoli, M., H. Tajik, B. Dalirnaghadeh \& F. Hariri, 2007. Prevalence of Linguatula serrata nymphs and gross changes of infected mesenteric lymph nodes in sheep in Urmia, Iran. Small Ruminant Research, 72, 73-76.

Yao, M. H., F. Wu \& L. F. Tang, 2008. Human pentastomiasis in China: Case report and literature review. The Journal of Parasitology, 94, 1295-1298.

Youssefi, M. R. \& S. H. Hadizadeh Moalem, 2010. Prevalence of Linguatula serrata nymphs in cattle in Babol Slaughterhouse, North of Iran. World Journal of Zoology, 5, 197-199.
Paper received 28.05.2016; accepted for publication 30.09.2016

\section{Correspondence:}

M. Hashemnia

Department of Pathobiology,

School of Veterinary Medicine, Razi University,

P.O. Box: 67156-85414, Kermanshah, Iran phone: +98-831-8322599-8329540

e-mail: m.hashemnia@razi.ac.ir 